\title{
Embedding auxetic properties in designing active-bending gridshells
}

\author{
Roberto Naboni \\ ACTLAB - ABC Department, Politecnico di Milano, \\ Milan, Italy \\ roberto.naboni@polimi.it \\ Stefano Sartori Pezzi \\ ACTLAB, Politecnico di Milano, \\ Milan, Italy \\ stefano.sartoripezzi@gmail.com
}

\begin{abstract}
Advancements in computational tools are offering designers the possibility to change their relationship with materials. The exploration of auxetic metamaterials, specifically engineered to obtain properties beyond those found in nature, is the promising field examined in this paper. The aim is to define tools and methods in order to design auxetics, and use them to create efficient active-bending structures. By programming their geometry through several parameters, it is possible to finely control curvature and structural resistance. The paper describes an original investigation into the process of programming such structures through the use of combined computational tools.
\end{abstract}

Keywords: Auxetics; Active-Bending; 3D Printing; Computational Design

\section{Introduction}

\section{From harvesting materials to metamaterials}

Advancements in computational tools are offering designers the possibility to radically change their relationship with materials. Typically, materials are harvested from the relatively limited amount present in nature and combined or adapted with standard procedures to fulfil our needs. The exploration of metamaterials, specifically engineered to obtain properties beyond the ones found in nature, is the promising field examined in this paper. In particular, the focus is on auxetics, known for their unique characteristic of becoming wider when stretched and narrower when compressed, as result of their negative Poisson's ratio (Evans, 1991). During the last decades, auxetic materials and structures have been examined by scientists and researches (Lakes,1987; Evans \& Alderson, 2000; Liu, 2006; Alderson, 2007; Liu \& Hu, 2010; Greaves et al. 2011). Fundamental properties of auxetics include: compressive strength and shear stiffness; enhanced indentation resistance; energy absorption; synclastic curvature when subject to a bending moment (Evans, 1990). The last one is the main property investigated in this paper with the objective of creating activebending gridshells, where highly specific curvatures can be achieved by the creation of custom auxetic patterns with variable geometrical features. The paper discusses the computationally-assisted design, form-finding and analysis, towards the introduction of a novel construction typology, following the idea of "material follows architecture".

\section{Active-bending with auxetics}

As the behaviour of auxetics is largely determined by their inherent geometry, this paper examines parameters and methods to pre-program two-dimensional auxetic patterns to realize active-bending structures with bespoke curvature and structural resistance. Previous work on the subject have involved the use of Particle Spring Systems (PSS) to simulate the form-finding of active-bending auxetic structures (Naboni \& Mirante, 2015). This method has the advantage of a high level of interaction during the design process, which requires the simplification of the geometries into a bidimensional description in order to reduce computation time. In contrast to previous approaches based on physics-based modelling environments, this paper proposes a more robust design and simulation method based on the large use of Finite Element Analysis. This is utilized in order to provide a complete mechanical description of the physical behaviour of such structures by including: mechanical material properties, variable cross-sections, interaction among individual components, nonlinear stress stiffening effects. Fundamental in the realization of active-bending structures is the selection of a suitable material. In fact, it needs to satisfy a specific ratio between flexural strength and flexural Young's modulus $(\sigma / E)$ greater than 2.5. When the material has a ratio greater than 10 it offers the opportunity to be used for elastic kinetic systems. The graph in Figure 1 highlights families of employable materials (Lienhard et al, 2013). 


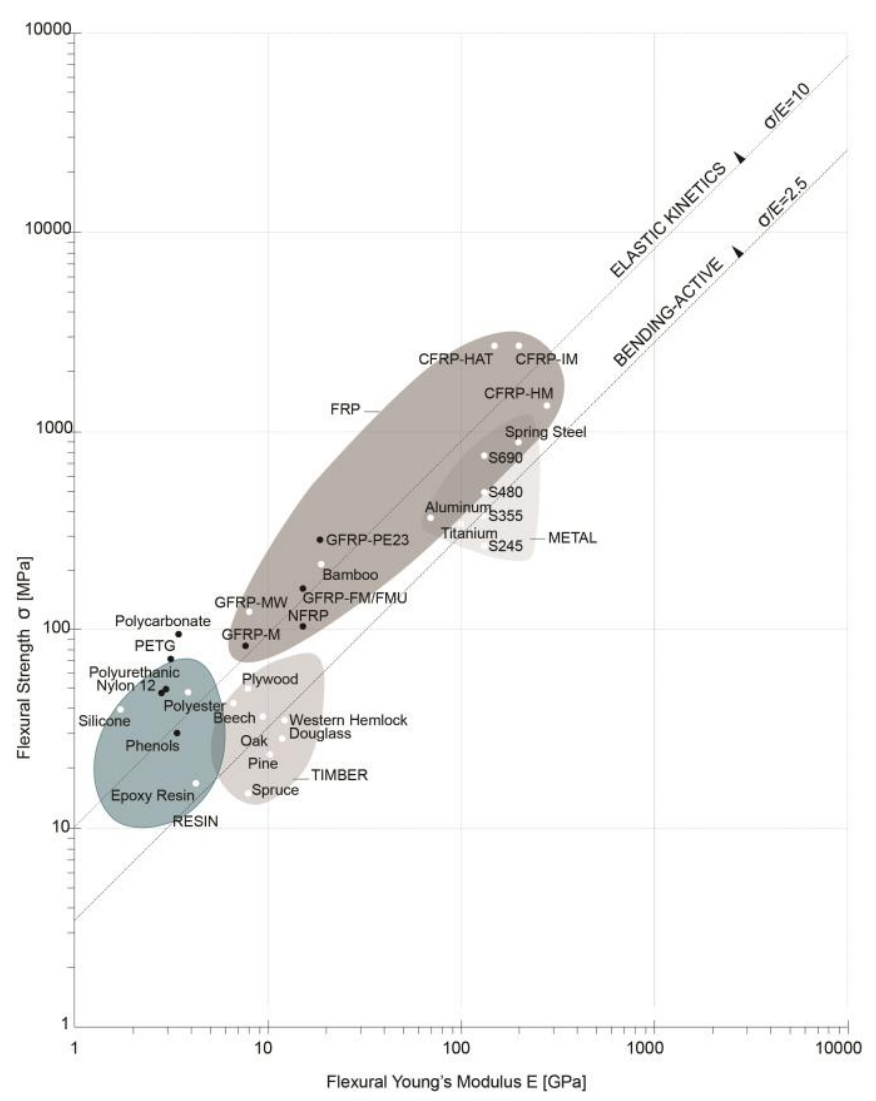

Figure 1: Ashby's diagram with comparison of suitable and unsuitable materials for bending-active structures (reproduced from Lienhard, J.,et al., 2013)

\section{Methodological Procedures}

The investigation of the behaviour of auxetic gridshells involve different phases, ranging from the general design and analysis of auxetic patterns to the discovery and tuning of the parameters influencing their behaviour. Generally, activebending structures are known for their challenging design and construction process, which has influenced their limited employment in spite of their advantageous structural efficiency. Nowadays, computer simulations ease the prediction of the elastic behaviour of such structures (Peloux, 2013). This research adopts a computational workflow encompassing parametric design, accurate mechanical analysis, and material prototyping.

\section{Parametric design of auxetic patterns}

Typical bi-dimensional auxetics are based on the periodic arrays of cells with a specific geometry, built on square and hexagonal grids. The parametric description of these patterns easily allow to control different topology and dimensional characteristics. A selection of well-known auxetic patterns have been designed to perform various tests. This includes hexagonal based patterns: Mesostructured Hexagonal, Triangular Hexagonal, Reentrant Honeycomb Hexagons; and square-based patterns: Sinusoidal Square, Chiral, Square Mesostructured.

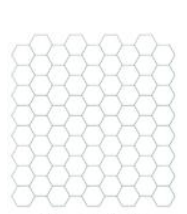

Hexagonal grid

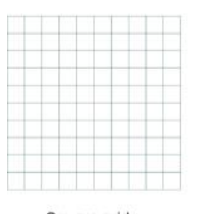

Square grid

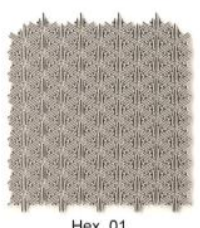

Hex_01

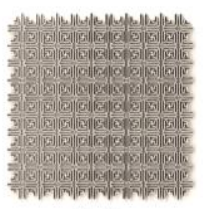

Sq_01

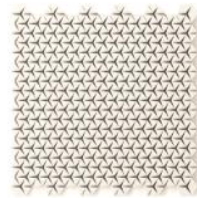

Hex_02

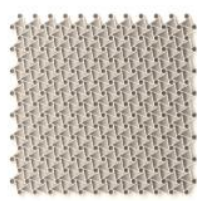

Sq_02

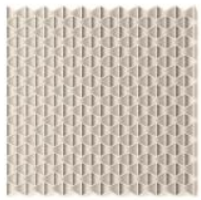

Hex_03

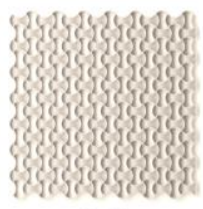

$\mathrm{Sq} 03$
Figure 2: Hexagonal-based patterns: (Hex_01) Hexagonal Mesostructured, (Hex_02) Triangular Hexagonal; (Hex_03) Reentrant Honeycomb Hexagons. Square-based patterns: (Sq_01) Squared Mesostructured (Sq_02) Triangular Chiral (Sq_03) Sinusoidal Square

\section{Comparative analysis of auxetic patterns}

The main mechanism to obtain the auxetic behavior in a material or structure is identified as the collective rotation of either nodal points or midpoints of struts (Körner \& LieboldRibeiro, 2014). The above-listed patterns follow this mechanism showing individual behaviour that requires a detailed observation. In this sense, a comparative analysis has been conducted with the goal of instrumentalizing their specific behaviour towards their use in active-bending structures. This analysis does not include the so called mesostructured patterns, which proved to be over demanding in terms of computation. Four types of structures, with an overall dimension of 20 by 20 meters, and sharing common strut sections of 0.5 meters height and 0.1 meters wide, have been compared in a numerical test performed with Ansys. All of the sample structures have been assigned with an isotropic polymeric material with ratio $\sigma / E=27,6$ This material description has convenient elastic properties for the in-plane compressive test of sample structures. Moreover, it can be easily tested in physical scaled prototypes produced with Additive Manufacturing (AM). This gives a fundamental parameter to understand more complex phenomena related to the auxetic behaviour, which are more pronounced as the Poisson's ratio tends to -1 . The elastic deformation of different specimens has been measured and analyzed to determine the Poisson's ratio for the two main directions, determined according to $V_{y x}=\Delta x / \Delta y$ with $x$ being the direction of uniaxial compression and $y$ the deformation obtained in the transverse direction.

The results provide evidence that certain patterns have an unequal response according to the axis of application of the forces. Consequently, we can categorize basic patterns in isotropic or anisotropic auxetics. Isotropic patterns include: Sinusoidal, Mesostructured, Square and Hexagonal Triangular; while anisotropic patterns consider: Hexagonal, Chiral Triangular and Square. In particular, the sinusoidal pattern has a typical two-way geometry which produces a perfectly isotropic behaviour (Figure 3) in contrast with other samples which have shown different degrees of anisotropic behaviour. A further analysis has been made to evaluate patterns in a bending test induced by the application of 
compressive distributed loads of $50 \mathrm{kN}$ from two parallel edges. In this setup, the curvature response useful to generate a active-bending gridshell is evaluated. Mesostructured patterns have shown a considerable flexural capacity, followed by the sinusoidal pattern which demonstrated a good balance of elasticity and flexural strength (Figure 4).

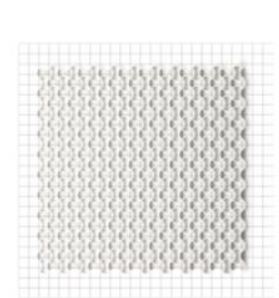

Undeformed Pattern

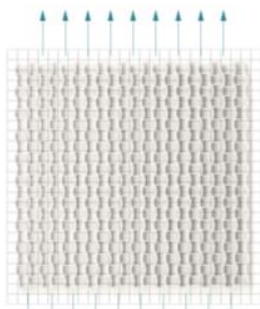

$, 1,1,1$, Elongated Pattern

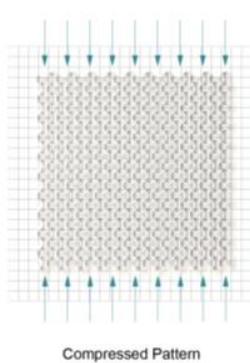

Compressed Pattem
Figure 3 Elongation and Compressive test on Sinusoidal Pattern

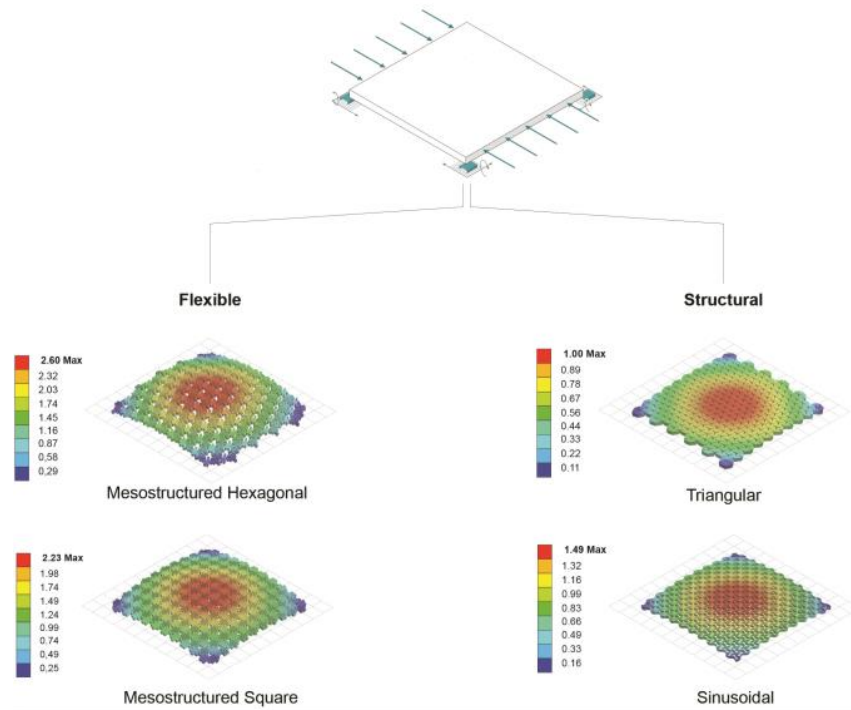

Figure 4: Bending behaviour of different patterns and comparison of their deformation

\section{Performative parameters of sinusoidal auxetics}

On the basis of the evidence from the comparative analysis, the sinusoidal pattern has been chosen for further studies as it has an isotropic behaviour, performs well in elastic bending and can be easily manufactured. Moreover, its specific layout allows for a straightforward investigation of its features and how these can be finely tuned to achieve explicit mechanical responses. The influence that each parameter has on the auxetic behaviour of a sinusoidal structure is inspected in the following analyses. This study is carried out on auxetic structures of 20 by 20 meters, with a rectangular strut section of $10 \mathrm{~cm}$ width by $100 \mathrm{~cm}$ height. Constant loading is applied horizontally from two parallel edges with an intensity of 50 $\mathrm{kN}$. Numerical analysis has been performed in order to comprehend the performance of sinusoidal auxetic structures with different erection methods and variations in concavity, cell dimension and cell height.

\section{Erection methods}

A fundamental aspect contributing to the definition of activebending auxetic structures is the configuration of the applied loads. Using a sinusoidal pattern, which presents auxetic behaviour in two axes, it is possible to investigate different static schemes. A dome-like shape with synclastic curvature can be obtained through the application of loads with a 45 degree inclination from the main axes. In the building field, these loads are applied on a structure with different techniques. From the literature review, a dozen completed active-bending structures have been selected in the past 50 years. Four main erection methods can be highlighted: (i) Push-up/Lift-up, (ii) Ease Down, (iii) Inflate, (iv) Compressive. Push-Up/Lift-Up uses towers and jacks to pull a partially constrained structure on the ground. (Figure 5a). Cables are often introduced in the system to join the grid gaps during the erection phase, in order to prevent structural collapse. The Ease Down method elevates the structure to its final height by the use of cranes, then the perimeter is pulled down to the ground. (Figure 5b) The use of pneumatic cushions, as formwork to lift the structure until completion, is an alternative with the advantage of homogeneous loads to avoid excessive local stresses in the phase of erection (Figure $5 \mathrm{c}$ ). The compressive technique buckles the structure by applying compressive loads from opposite edges/corners. The LiftUp/Push-Up method is likely to cause overstressing during erection, while Ease Down and Inflate are the most geometrically precise and the least structurally demanding (Quinn and Gengnagel, 2014).
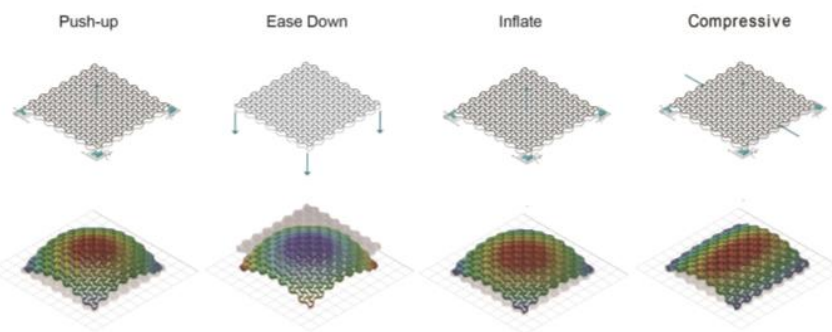

Figure 5: Comparison between different Erection method, on Sinusoidal Pattern, subjected at the same intensity load varying the construction system. From left to right, Push-up; Ease Down; Inflate, Compressive

\section{Concavity parameter}

By analyzing the layout of the sinusoidal pattern, it is intuitive to understand that the concave edges behave as hinge regions. To better understand the impact of this parameter, three sample structures with different concavity parameters are designed and tested with bending induced by compressive loads applied to opposite edges. Variations in this parameter substantially affects the gaussian curvature of the structure. The typical sinusoidal configuration has a concavity parameter $\mathrm{C}_{p}=+1$, which conforms as a anticlastic structure, with positive gaussian curvature (Figure 6a). A gradual reduction of this parameter generates different curvature outputs. When $\mathrm{C}_{p}=0$, the sample structure behaves 
as a developable surface with gaussian curvature $\mathrm{K}=0$ (Figure 6b). A convex pattern $\mathrm{C}_{p}=-1$ generates a sort of elliptic alternated pattern, which confers an anticlastic curvature $\mathrm{K}=-$ 1. These results provide confirmatory evidence that the concavity parameter influences the Poisson's ratio of a pattern.
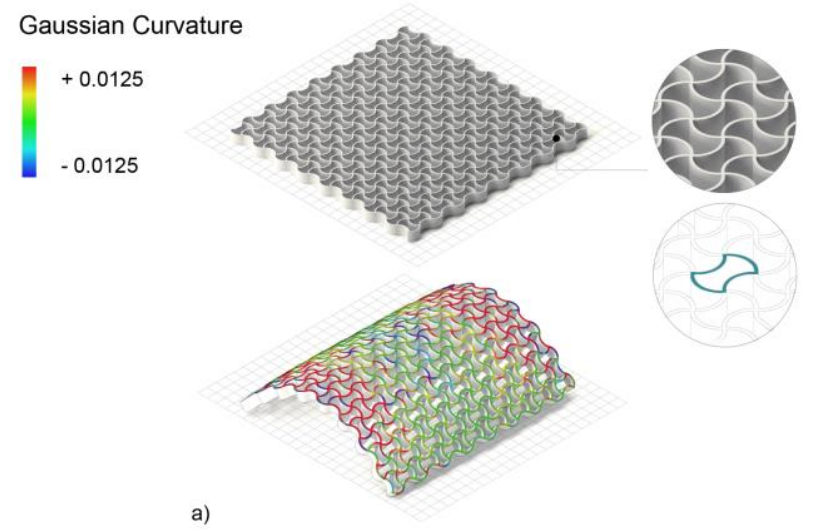

a)

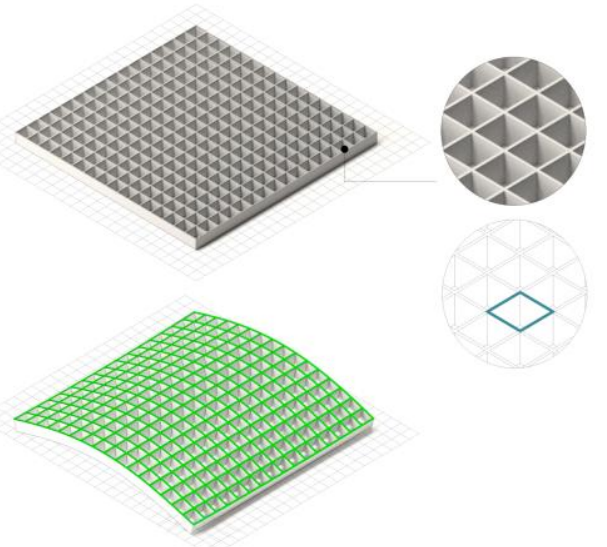

b)

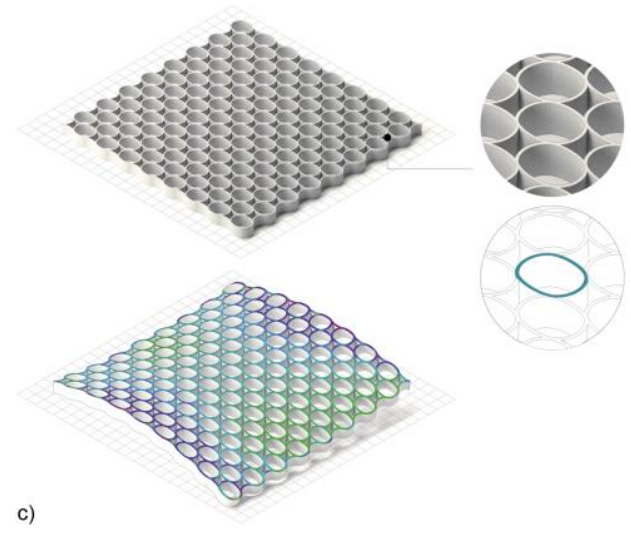

Figure 6: Analysis of the gaussian curvature for patterns with different concavity. $\mathrm{Cp}=+1$ (a), $\mathrm{Cp}=0$ (b), $\mathrm{Cp}=0$ (c)

\section{Struts section}

Another fundamental parameter is related to the sectional height of the struts composing the auxetic structure.

Variations in this parameter influence the overall bending capacity by influencing the moment of inertia. Higher values increase the flexural strength of the structure. This behavior is measured with multiple sectional heights (i) 1 meter, (ii) 0.5 meters, (iii) 0.1 meters, and the respective bending curvature are confronted (Figure 7). It is observed that small sectional heights tend to create developable surfaces in bending, while larger heights produce more prominent curvatures, in both the synclastic and anticlastic configurations.

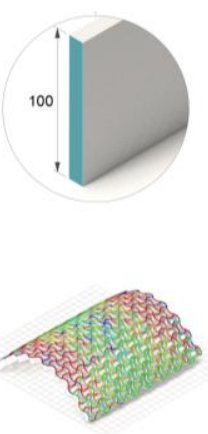

a)

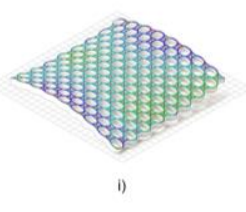

Figure 7: Different curvature tuning the height of the cross section: a) $10 \times 100[\mathrm{~cm}]$; b) $10 \times 50[\mathrm{~cm}]$; c) $10 \times 10[\mathrm{~cm}]$

\section{Dimension of the base unit}

The dimension of the base pattern unit is another fundamental parameter. Two samples with different unit dimensions have have been compared in a bending test. The first one is with a basic cell of 1.40 meters, and the second one with a dimension of 0.70 meters. The test highlights how the second option, with double higher density, offers more bending resistance, but does not affect the gaussian curvature (Figure 8). 

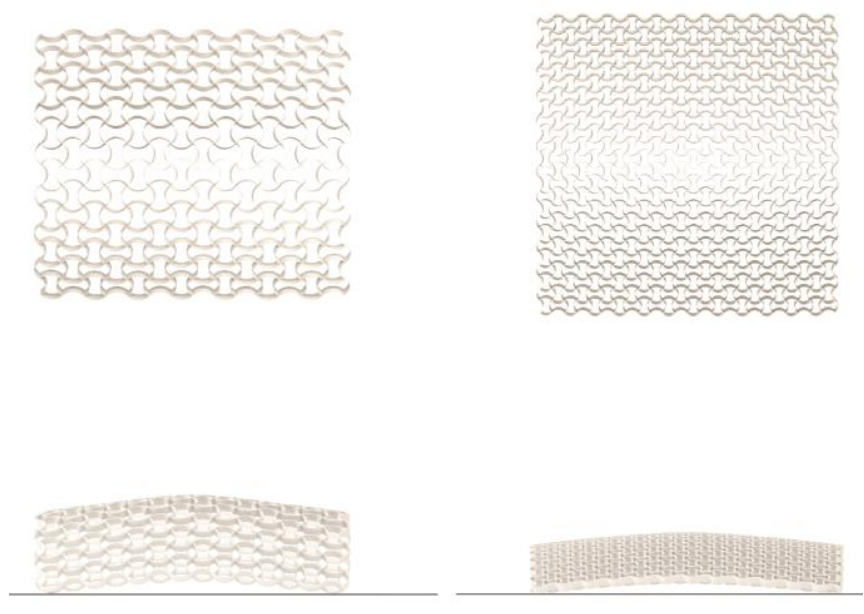

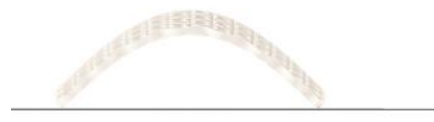

a)

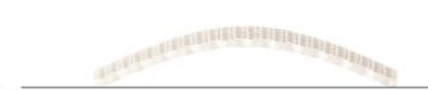

Figure 8: Comparison between the different pattern dimension. a) Density variation grid $14 \times 14$ b) Density variation grid $28 \times 28$

\section{Prototyping of auxetics}

In parallel with numerical tests, a set of scaled models has been produced to offer the possibility of a direct interaction and develop an intuitive understanding of the auxetic behaviour. The prototypes are produced by means of Fused Deposition Modelling (FDM), a technique which allows the direct manufacturing of their shape, and the flexibility to use materials with different characteristics. Two samples structures have been manufactured in order to compare the variation induced by the use of different Concavity Parameters (Cp): positive concavity parameter in two directions $C_{p}=1$ (Figure 9a), and negative concavity $C_{p}=-1$ in one direction (Figure 9b). Both patterns are built on the base of a squared grid with overall dimensions of 200 by 200 millimeters, base unit with dimensions of 10,25 by 10,25 millimeters, and strut cross section $1 \mathrm{~mm}$ by 2 millimeters (width/height). While geometrical characteristics can be easily scaled, the definition of a proper material in this scale requires different trials to match the prevision of the simulated structure in real scale ( 20 by 20 meters). The final choice was the use of a Thermoplastic Elastomer (TPE) with flexural modulus $\mathrm{E}_{\text {bend }}=32 \mathrm{MPa}$. The two samples have been loaded with compressive forces with a $45^{\circ}$ orientation from two opposite corners, and highlighted two distinct gaussian curvature: purely synclastic (Figure 10a) and anticlastic (Figure 10b), substantially confirming the prediction of the test developed previously.

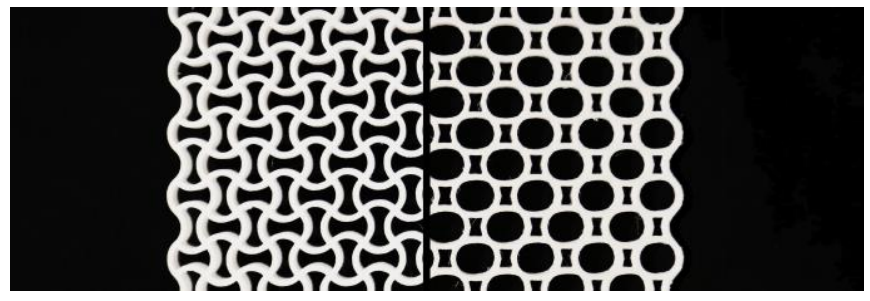

Figure 9: Top view of two different prototype samples with different concavity parameter: $\mathrm{Cp}=+1$ (Figure 9a), $\mathrm{Cp}=-1$ (Figure 9b)

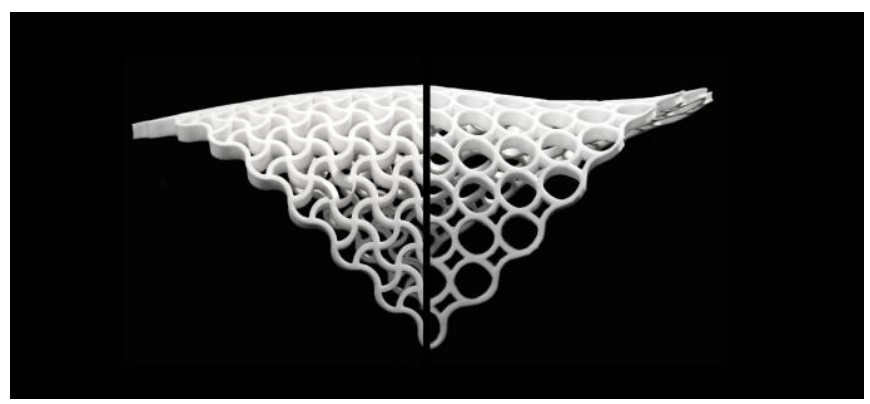

Figure 10: 3D printed bent prototypes highlight a different gaussian curvature: synclastic (Figure 10a) and anticlastic (Figure 10b)

\section{Results}

The research focused on the programming and analysis of auxetics towards their use in active-bending structures. A computational workflow that combines parametric design with Grasshopper and FEM analysis with Ansys is proposed to accurately test and measure the elastic behaviour of these structures. With this methodology different patterns are analyzed. Results show that the sinusoidal pattern is completely isotropic, has the lowest value of Poisson's Ratio $(-0.67)$ among the tested samples, and performs well in elastic bending (Figure 3-4). This data, along with considerations on its manufacturability, supported the decision for further dedicated analyses. These have highlighted how the sinusoidal pattern can be tuned through the parametrization of its geometrical characteristics. In particular, changes in the concavity of the pattern directly affects the Poisson's Ratio thus allowing for regulations of the gaussian curvature of an elastically bent structure (Figure 6). The parametrization can happen independently on the two main axes. Variations in the sectional height of the structure influences the flexural strength as well as the gaussian curvature, as overly thin sections produce developable surfaces once they are bent (Figure 7). The dimension of the base unit of auxetics impacts the resistance to bending, but does not affect the gaussian curvature (Figure 8). According to these results, two samples structures are designed and prototyped in scaled physical models to validate the numerical tests. The comparison shows how gridshells with different gaussian curvatures (Figure 11) can be achieved with variation to the Concavity parameters and given the same set of boundary conditions (Figure 10) and anticlastic curvature (Figure 11). The comparison of these structures confirms the results of the parameters analysis. 


\section{Discussion}

The use of computational tools for the design and analysis of complex constructions extends the understanding of material behaviour. In the past years the advanced customization of architectural design and construction has allowed designers to push the study of materials, structures and forms, extending the boundary of architecture towards highperformance systems (Naboni \& Paoletti, 2015). In this perspective, the study of metamaterials, such as the auxetics, allow us to proceed top-down, from the definition of certain architectural performances to the programming and embedding of specific material features. In this perspective, this paper describes a study which attempts to define a procedure for the evaluation of parameters and behaviours of active-bending auxetic structures. A successful method is found in the extensive use of FEM coupled with prototyping of scaled models. The combination of the two approaches deepens the understanding of auxetics towards their use in active-bending structures: physical models provide an intuitive understanding of the material, while numerical analysis contributes to scale the approach into large structures. This approach proves to be accurate but timeconsuming, thus ideal for the purpose of analytic studies. The sinusoidal pattern emerges as an interesting option for the use in architecture. This has a relative simple geometry and is purely isotropic, thus allowing for complex configurations of anchor points and, as consequence, higher freedom in the design of complex spatial articulations. Fundamental parameters for highly specific pre-programming of these structures are found and quantified for a structure of 20 by 20 meters. Dimensional references for these parameters are provided for the realization of a case study structure in composite materials. Further development could involve the development of more intuitive and interactive tools to compute such complex material behaviour with sufficient precision, along with the definition of large-scale manufacturing techniques for seamless structures.

\section{Acknowledgements}

We would like to thank Anja Kunic and Maia Zheliazkova for the ideas and support in the preparation of the contents of this paper. We also express our gratitude to KLONER 3D which provided the fundamental fabrication equipment to develop this work.

\section{References}

Alderson, A. \& Alderson, K. L. (2007). Auxetic materials, Special issue paper in Proc. IMechE, Vol. 221 Part G: J. Aerospace, 27 February.

Du Peloux, L., Baverel, O., Caron, J. F. \& Tayeb, F. (2015). From shape to shell: a design tool to materialize freeform shapes using gridshell structures, in Design Modelling Symposium, Berlin.

Evans, K. E. \& Alderson, A. (2000). Auxetic Materials: Functional Materials and Structures from Lateral Thinking, in Advanced Materials, 12, No.9, 617-628.

Evans, K. (1990). Tailoring the negative Poisson ratio. Chem. Ind.London 20, 654-657.
Fleischmann, M. \& Menges, A. (2012). Physics-Based Modeling as an Alternative Approach to Geometrical Constraint - Modeling for the Design of Elastically Deformable Material Systems, in Simulation, Prediction and Evaluation, Volume 1, pp 565-576.

Happold, E. \& Liddell, W. I. (1975). Timber lattice roof for the Mannheim Bundesgartenschau, in The Structural Engineer, Vol. 53. (No.3).

Kelly, O. J., Harris, R. J. L., Dickson, M. G. T. \& Rowe, J. A. (2001). Construction of the Downland Gridshell, in The Structural Engineer, 79, No. 17, 4 September.

Körner, C. and Liebold-Ribeiro, Y., (2014), A systematic approach to identify cellular auxetic materials, in Smart Mater. Struct. 24 025013 (10pp), 19 December.

Lafuente, E., Gengnagel, C., Sechelmann, S. \& Rörig, T. (2015). On the Materiality and Structural Behaviour of highly-elastic Gridshell Structures; in Research of Paper Department of Architecture, University of the Arts, Berlin, Germany, May 06.

Lakes, R. S. (1987). Foam structure with a negative Poisson's Ratio, in Science, Vol. 235.

Lienhard, J., Alpermann, H., Gengnagel, C. \& Knippers, J. (2013). Active Bending, A Review on Structures where Bending is used as a Self-Formation Process, in "International Journal of Space Structures" Volume 28, Number $3 \& 4$.

Liu, Y. \& Hu, H. (2010) A review on auxetic structures and polymeric materials, Scientific Research and Essays Vol. 5 (10), pp. 10521063, 18 May.

Liu,Q. (2006). Literature Review: Materials with Negative Poisson's Ratios and Potential Applications to Aerospace and Defence. (Electronic Thesis or Dissertation). Retrieved from item: http://dspace.dsto.defence.gov.au/dspace/handle/1947/4436.

Mir, M., Najabat Ali, M., Sami, J. \& Ansar, U. (2014). Review of Mechanics and Applications of Auxetic Structure, in Hindawi Publishing Corporation, Advances in Materials Science and Engineering.

Mozaffar, S. R. \& Zaini, A. (2015). Finite Element Modelling of Different 2D Re-Entrant Structures of Auxetic Materials, in Proc. of the Second Intl. Conf. on Advances in Mechanical and Automation Engineering.

Naboni, R. \& Mirante, L. (2015). Metamaterial computation and fabrication of auxetic patterns for architecture. Blucher Design Proceedings, 2(3), 129-136.

Naboni, R \& Paoletti, I. (2015). Advanced Customization in Architectural Design and Construction. Springer International Publishing.

Pone, S., Colabella, S., D'Amico, B., Fiore, A., Lancia, D. \& Parenti, B. (2013). Timber Post-formed Gridshell: Digital Form-finding / drawing and building tool, Proceedings of the International Association for Shell and Spatial Structures (IASS) Symposium 2013, Beyond the limits of man, 23-27 September.

Pone, S., Colabella, S., Parenti, B., Lancia, D. \& Fiore, A. (2013). Construction and form-finding of a post-formed timber grid-shell, in Structures and Architecture: Concepts, Applications and Challenges, Cruz (ed) Taylor \& Francis Group, London. 
SIGraDi 2016, XX Congress of the Iberoamerican Society of Digital Graphics

9-11, November, 2016 - Buenos Aires, Argentina

Quinn, G., Gengnagel, C. (2014). A review of elastic gridshells, their erection methods and the potential use of pneumatic formwork, in Mobile and Rapidly Assembled Structures, WIT Transactions on The Built Environment, Vol 136 pp 129-143.

Schleicher, S., Rastetter, A., La Magna, R. A., Schönbrunner, A., Haberbosch, N. \& Knippers,J. (2015). Form Finding and design potentials of bending-Active Plate structures, in Modelling Behaviour, Springer International Publishing Switzerland.

Sun, Y. \& Pugno, N. M. (2013). In plane stiffness of multifunctional hierarchical honeycombs with negative Poisson's ratio substructures, in Composite Structures, Number 106, pp 681-689. 